UR-1589

$\mathrm{ER} / 40685 / 939$

hep-ph/9911536

November 1999

\title{
Vector Boson Transverse Momentum Distributions at the Tevatron
}

\author{
G. Corcella团 \\ Department of Physics and Astronomy, University of Rochester, \\ Rochester, NY 14627, U.S.A.
}

\begin{abstract}
M.H. Seymour
Rutherford Appleton Laboratory, Chilton, Didcot, Oxfordshire. OX11 0QX. U.K.
\end{abstract}

\begin{abstract}
We show vector boson transverse momentum distributions at the Tevatron, obtained by running the HERWIG Monte Carlo event generator with matrixelement corrections. We compare our results with some recent D $\varnothing$ and CDF data.
\end{abstract}

Vector boson production at hadron colliders is a fundamental process to test Quantum Chromodynamics and the Standard Model of the electroweak interactions. The lowest order processes $q \bar{q}^{\prime} \rightarrow W$ and $q \bar{q} \rightarrow Z / \gamma^{*}$ are not sufficient to perform reliable phenomenological predictions, but the initial-state radiation has to be taken into account. A possible way to deal with such multiple emissions consists in running a Monte Carlo event generator. Standard Monte Carlo algorithms [1] describe parton cascades in the soft/collinear approximation, with 'dead zones' in the phase space which can be filled by the using of the exact first-order matrix element.

In [2] we implemented matrix-element corrections to the HERWIG simulation of Drell-Yan interactions: we filled the missing phase space using the exact $\mathcal{O}\left(\alpha_{S}\right)$ matrix element (hard corrections) and corrected the shower in the already-populated region using the exact amplitude for every hardest-so-far emission (soft corrections). For $W$ production at the Tevatron, about $4 \%$ of the events are generated in the dead zone, about half of which are $q \bar{q}^{\prime} \rightarrow W g$ events. Similar results hold for $Z$ production as well.

An interesting observable to study is the vector boson transverse momentum $q_{T}$, which is the object of many theoretical and experimental analyses. While in the parton shower approximation it has to be $q_{T}<m_{W, Z}$, after matrix-element corrections a fraction of events with larger values of $q_{T}$ is to be expected. In Fig. 1 (a), we plot the $W q_{T}$ $\ddagger$ Talk given at the UK Phenomenology Workshop on Collider Physics, Durham, U.K., 19-24 September 1999. 

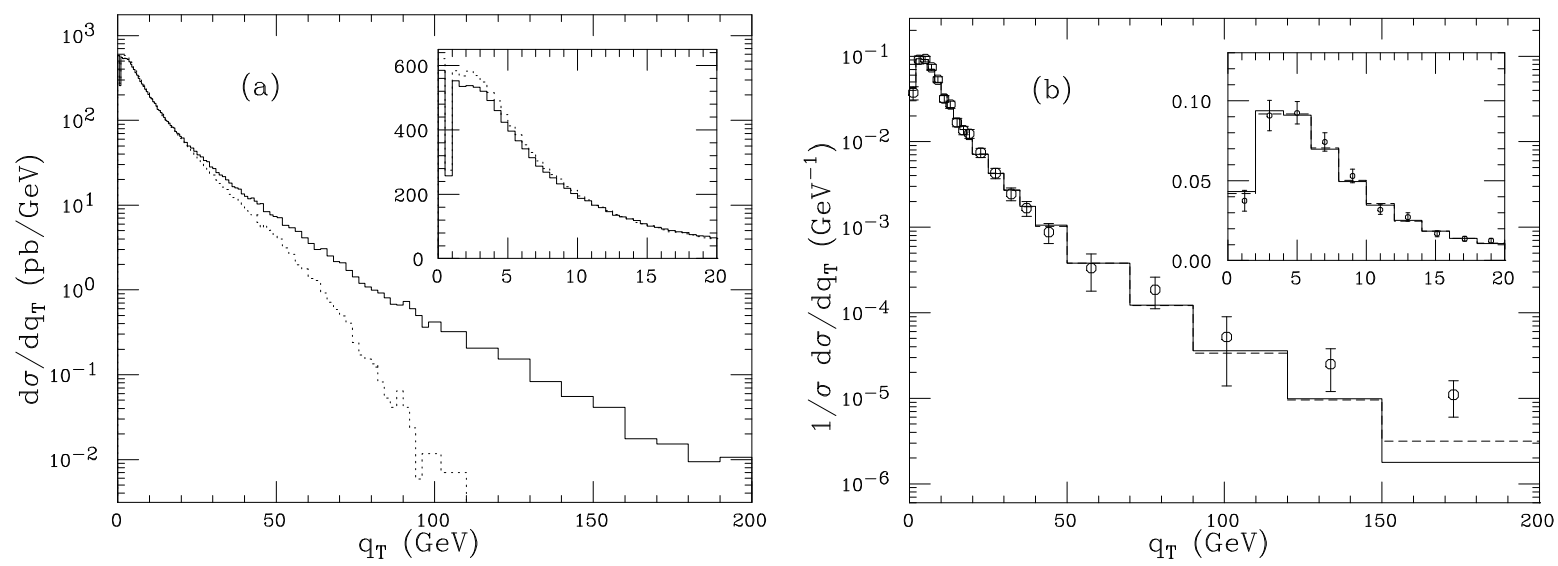

Figure 1. (a): $W$ transverse momentum distribution at the Tevatron according to HERWIG before (dotted line) and after matrix-element corrections (solid) for $q_{T \text { int }}=0$; (b): comparison of the DØ data with HERWIG 6.1 after detector corrections for $q_{\text {Tint }}=0$ (solid) and $1 \mathrm{GeV}$ (dashed).

spectrum at the Tevatron according to HERWIG 5.9, the latest public version, and to HERWIG 6.1, the new version including matrix-element corrections to Drell-Yan processes. HERWIG has the option to vary the intrinsic transverse momentum $q_{\text {Tint }}$ of the incoming partons, which is set to zero, its default value, in the distributions shown in Fig. 1 (a). We observe a remarkable impact of the corrections: after some $q_{T}$ the 5.9 version does not give events anymore, while HERWIG 6.1 still has some events generated via the exact matrix element. In Fig. 1 (b) we compare some recent D $\varnothing$ data [3] on $W$ production at the Tevatron with the HERWIG 6.1 results, which we corrected in order to take the detector smearing effects into account. We find good agreement overall; we also consider the option of $q_{\text {Tint }}=1 \mathrm{GeV}$, but do not see any relevant effect after the detector corrections.

As far as $Z$ production is concerned, we have some preliminary CDF data [4], already corrected for detector effects, which we compare with HERWIG 6.1 in Fig. Q, where the options $q_{T \text { int }}=0,1$ and $2 \mathrm{GeV}$ are investigated. The agreement is acceptable and the role of the implemented matrix-element corrections is crucial in order to succeed in fitting in with the data for $q_{T}>50 \mathrm{GeV}$. At very low $q_{T}$, the best fit is obtained by setting $q_{T \text { int }}=2 \mathrm{GeV}$. In Fig. [3, we plot the ratio of the $W$ and the $Z$ transverse momentum spectra, both normalized to unity, for different values of $q_{T \text { int }}$. Although it can be seen from Fig. 2 (b) that the $Z q_{T}$ spectrum depends strongly on $q_{T \text { int }}$ at low $q_{T}$, the ratio of the $W$ and $Z$ spectra is insensitive to it. This is good news for the $W$ mass measurement in hadron collisions, as this ratio is one of the main theory inputs that is needed. A strong dependence on unknown non-perturbative parameters like $q_{T \text { int }}$ could limit the accuracy of the $W$ mass measurement at the Tevatron and, ultimately, at the LHC.

We have added matrix-element corrections to HERWIG's treatment of vector boson production in hadron collisions. They make an enormous difference at high transverse 

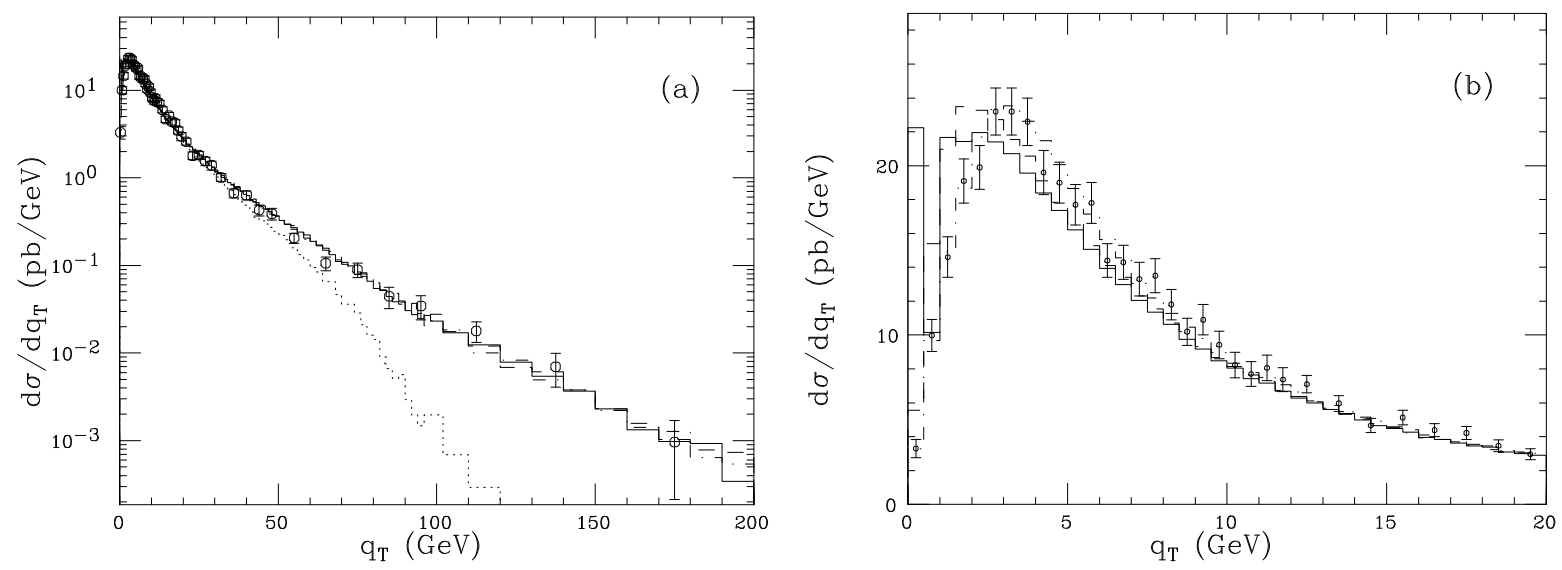

Figure 2. $Z$ transverse momentum distribution according to HERWIG 5.9 with zero intrinsic transverse momentum (dotted line) and according to HERWIG 6.1 with $q_{T \text { int }}=0$ (solid), $1 \mathrm{GeV}$ (dashed) and $2 \mathrm{GeV}$ (dot-dashed), compared with the CDF data over the whole spectrum (a) and for low $q_{T}$ values (b).

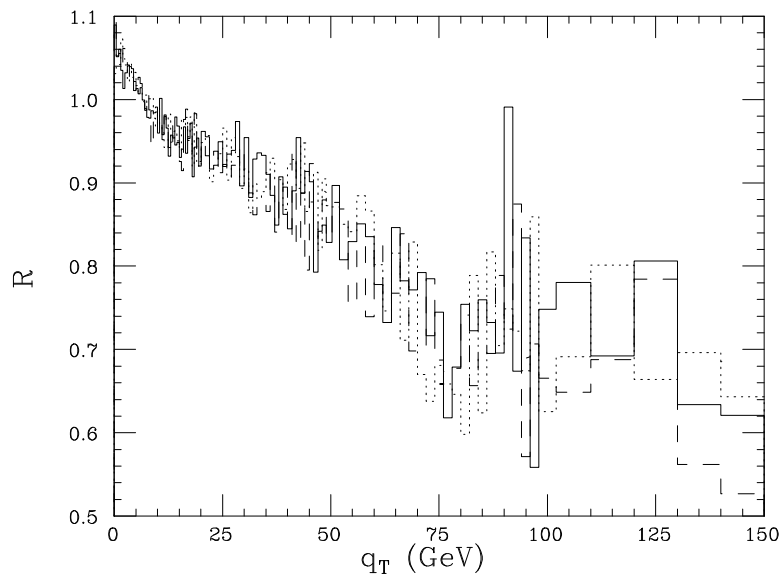

Figure 3. The ratio $R$ of the $W$ and $Z$ transverse momentum spectra, running HERWIG 6.1, for $q_{\text {Tint }}=0$ (solid), $1 \mathrm{GeV}$ (dashes) and $2 \mathrm{GeV}$ (dotted).

momentum $q_{T}$, but little at low $q_{T}$. Although the dependence of the results on the non-perturbative intrinsic $q_{T}$ of partons in the proton $\left(q_{T \text { int }}\right)$ is quite strong at low $q_{T}$, it is very similar in the $W$ and $Z$ cases, so that the ratio of the two $q_{T}$ spectra is almost independent of $q_{T \text { int }}$.

\section{References}

[1] G. Marchesini et al., Comput. Phys. Commun. 67 (1992) 465.

[2] G. Corcella and M.H. Seymour, hep-ph/9908335.

[3] DØ Collaboration, B. Abbott et al., Phys. Rev. Lett. 80 (1998) 5498.

[4] CDF Collaboration, T. Affolder et al., Fermilab-Pub-99/220-E. 